CRYSTALLOGRAPHIC COMMUNICATIONS

ISSN 2056-9890

Received 6 September 2021

Accepted 20 September 2021

Edited by W. T. A. Harrison, University of Aberdeen, Scotland

Keywords: crystal structure; 1,2,3-triazole; amide; 1,2,3-triazole-4-carboxamide; Hirshfeld surface analysis.

CCDC reference: 736128

Supporting information: this article has supporting information at journals.iucr.org/e

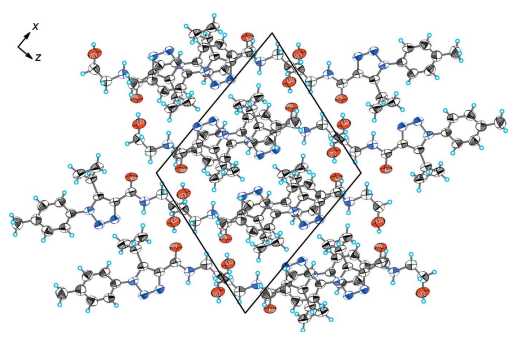

OPEN $\odot$ ACCESS

\section{Synthesis, crystal structure and Hirshfeld surface analysis of 5-cyclopropyl-N-(2-hydroxyethyl)-1-(4- methylphenyl)-1H-1,2,3-triazole-4-carboxamide}

\author{
Nazariy T. Pokhodylo, ${ }^{a *}$ Yurii Slyvka ${ }^{b}$ and Volodymyr Pavlyuk ${ }^{b}$ \\ a Department of Organic Chemistry, Ivan Franko National University of Lviv, Kyryla i Mefodiya, 6, Lviv, 79005, Ukraine, \\ and ${ }^{\mathbf{b}}$ Department of Inorganic Chemistry, Ivan Franko National University of Lviv, Kyryla i Mefodiya, 6, Lviv, 79005, \\ Ukraine. ${ }^{*}$ Correspondence e-mail: pokhodylo@gmail.com
}

The title compound, $\mathrm{C}_{15} \mathrm{H}_{18} \mathrm{~N}_{4} \mathrm{O}_{2}$, was obtained via a two-step synthesis (Dimroth reaction and amidation) for anticancer activity screening and was selected from a $1 H$-1,2,3-triazole-4-carboxamide library. The cyclopropyl ring is oriented almost perpendicular to the benzene ring [dihedral angle $=87.9(1)^{\circ}$ ], while the dihedral angle between the mean plane of the cyclopropyl ring and that of the triazole ring is $55.6(1)^{\circ}$. In the crystal, the molecules are linked by $\mathrm{O}-\mathrm{H} \cdots \mathrm{O}$ and $\mathrm{C}-\mathrm{H} \cdots \mathrm{N}$ interactions into infinite ribbons propagating in the [001] direction, which are interconnected by weak $\mathrm{C}-\mathrm{H} \cdots \mathrm{O}$ interactions into layers. The intermolecular interactions were characterized via Hirshfeld surface analysis, which indicated that the largest fingerprint contact percentages are $\mathrm{H} \cdots \mathrm{H}(55.5 \%), \mathrm{N} \cdots \mathrm{H} / \mathrm{H} \cdots \mathrm{N}(15.4 \%), \mathrm{C} \cdots \mathrm{H} / \mathrm{H} \cdots \mathrm{C}(13.2 \%)$ and $\mathrm{O} \cdots \mathrm{H} /$ H. . O $(12.9 \%)$.

\section{Chemical context}

The 1,2,3-triazolyl-4-carboxamide motif is of great interest in drug discovery, especially in relation to anticancer and antimicrobial research. Besides the well-known drugs rufinamide and carboxyamidotriazole, several preclinical studies are ongoing. As an example of antitumour activity evaluations, libraries of 1,2,3-triazole-4-carboxamides containing podophyllotoxin (Reddy et al., 2018), 1- $R-N-[(1-R-1 H-1,2,3$-triazol4-yl)methyl]-1H-1,2,3-triazole-4-carboxamides (Elamari et al., 2013), 5-(trifluoromethyl)-1H-1,2,3-triazole-4-carboxamides (Wang et al., 2018; Zhou et al., 2014) and 1-benzyl- $\mathrm{N}$-[2(phenylamino)pyridin-3-yl]-1H-1,2,3-triazole-4-carboxamides (Prasad et al., 2019) have been tested. Several 1,4,5-trisubstituted 1,2,3-triazole-4-carboxamides showed high affinity in the nanomolar concentration range toward Hsp90 associated with cell proliferation inhibition (Taddei et al., 2014; Giannini et al., 2015). Moreover, 4-[4-(hydrazinecarbonyl)-5methyl-1H-1,2,3-triazol-1-yl]benzenesulfonamide was found to act as a COX-2 inhibitor (Bekheit et al., 2021).

In our previous studies, new active compounds with a 1,2,3triazolyl-4-carboxamide motif were reported (Shyyka et al., 2019; Pokhodylo, Shyyka, Finiuk \& Stoika, 2020; Pokhodylo, Slyvka \& Pavlyuk, 2020). Additionally, 1,2,3-triazolyl-4carboxamide derivatives were found to be inhibitors of the Wnt/ $\beta$-catenin signalling pathway (Obianom et al., 2019). In addition, compounds with this motif exhibited fungicidal (Wang et al., 2014), antiviral (Krajczyk et al., 2014) and antimicrobial (Pokhodylo et al., 2021; Jadhav et al., 2017) activities. 
The most convenient synthetic path to diverse $1 H$-1,2,3-triazole-4-carboxamides is a two-step synthesis involving the Dimroth reaction of organic azides with $\beta$-ketoesters (Pokhodylo \& Obushak, 2019) followed by amidation of the resulting $1 H-1,2,3$-triazole-4-carboxylic acids.

Given the practical interest of 1-aryl-1H-1,2,3-triazole-4carboxamides in anticancer and antimicrobial research, in the present paper, we report the molecular and crystal structure of the title compound $\mathrm{C}_{15} \mathrm{H}_{18} \mathrm{~N}_{4} \mathrm{O}_{2}$, highlighting its molecular conformation and analysing the intermolecular interactions. The cyclopropyl substituent was selected as it meets the criteria of lead-oriented synthesis, increasing the number of $s p^{3}$-carbon atoms, but at the same time is conformationally restricted and occupies minimal volume among other C3-alkyl substituents. Moreover, the 5-cyclopropyltriazole fragment could appear as a bisected or perpendicular conformer.<smiles>Cc1ccc(-n2nnc(C(=O)NCCO)c2C2CC2)cc1</smiles>

\section{Structural commentary}

The title compound crystallizes in the monoclinic centrosymmetric space group $P 2_{1} / c$, with one molecule in the asymmetric unit as shown in Fig. 1. The molecular structure possesses three conformational degrees of freedom due to free rotation about the $\mathrm{C} 9-\mathrm{C} 10, \mathrm{C} 8-\mathrm{C} 11$ and $\mathrm{N} 1-\mathrm{C} 1$ single bonds. The $\mathrm{C} 10 / \mathrm{N} 4 / \mathrm{O} 1$ amide group is turned slightly relative to the $\mathrm{N} 1 /$ $\mathrm{N} 2 / \mathrm{N} 3 / \mathrm{C} 8 / \mathrm{C} 9$ triazole ring by $11.71(4)^{\circ}$. Within the $\mathrm{C} 11 / \mathrm{C} 12 /$ $\mathrm{C} 13$ cyclopropyl ring, the $\mathrm{C}-\mathrm{C}$ bond lengths differ by an insignificant amount $[\mathrm{C} 11-\mathrm{C} 12=1.488(3), \mathrm{C} 11-\mathrm{C} 13=$ 1.492 (3), $\mathrm{C} 12-\mathrm{C} 13=1.471$ (3) $\AA$ ] . The cyclopropyl ring is oriented almost perpendicular to the $\mathrm{C} 1-\mathrm{C} 6$ benzene ring and the dihedral angle between these planes is $87.9(1)^{\circ}$. The

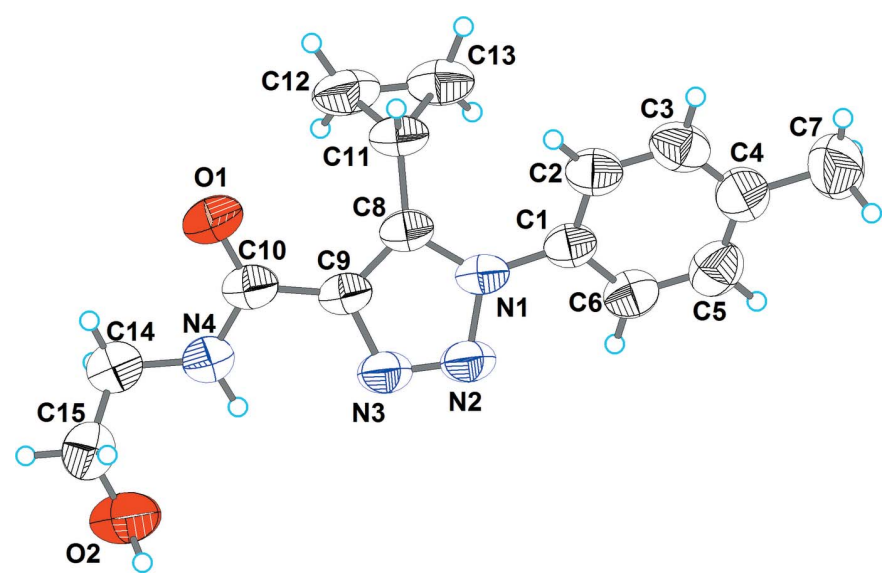

Figure 1

The molecular structure of the title compound with displacement ellipsoids drawn at the $50 \%$ probability level.
Table 1

Hydrogen-bond geometry $\left(\AA{ }^{\circ}\right)$.

\begin{tabular}{lllll}
\hline$D-\mathrm{H} \cdots A$ & $D-\mathrm{H}$ & $\mathrm{H} \cdots A$ & $D \cdots A$ & $D-\mathrm{H} \cdots A$ \\
\hline $\mathrm{O} 2-\mathrm{H} 2 \cdots \mathrm{O} 1^{\mathrm{i}}$ & $0.95(3)$ & $1.78(3)$ & $2.734(2)$ & $177(3)$ \\
$\mathrm{C} 11-\mathrm{H} 11 \cdots \mathrm{N3}^{\mathrm{ii}}$ & 0.98 & 2.61 & $3.391(2)$ & 137 \\
$\mathrm{C} 5-\mathrm{H} 5 \cdots \mathrm{O} 2^{\mathrm{iii}}$ & 0.93 & 2.66 & $3.564(3)$ & 164 \\
\hline
\end{tabular}

Symmetry codes: (i) $x,-y+\frac{1}{2}, z-\frac{1}{2}$; (ii) $x,-y+\frac{1}{2}, z+\frac{1}{2}$; (iii) $-x+1,-y+1,-z+1$.

dihedral angle between the mean plane of the cyclopropyl ring and that of the triazole ring is $55.6(1)^{\circ}$.

A similar location of the cyclopropyl ring relative to the 1,2,3-triazole ring was also observed in 5-cyclopropyl-1-(3methoxyphenyl)-1H-1,2,3-triazole-4-carboxylic acid (Pokhodylo et al., 2017), but in the structure of the related compound $\mathrm{N}$-(4-chlorophenyl)-5-cyclopropyl-1-(4-methoxyphenyl)- $1 \mathrm{H}$ 1,2,3-triazole-4-carboxamide (Pokhodylo \& Slyvka et al., 2020), the cyclopropyl ring is close to coplanar with the aryl substituent. An intramolecular $\mathrm{N} 4-\mathrm{H} 4 \cdots \mathrm{N} 3$ close contact $\left(\mathrm{H} \cdots \mathrm{N}=2.37 \AA ; \mathrm{N}-\mathrm{H} \cdots \mathrm{N}=106^{\circ}\right)$ is observed.

The dihedral angle between the tolyl and 1,2,3-triazole rings in the title compound is $32.75(7)^{\circ}$, which is comparable with the corresponding angle in 5-cyclopropyl-1-(3-methoxyphenyl)-1 $H-1,2,3$-triazole-4-carboxylic acid [39.1 (2) ${ }^{\circ}$ but lower than in the structure of 5-methyl-1-(4-nitrophenyl)- $1 H$-1,2,3triazol-4-ylphosphonate $\left[45.36(6)^{\circ}\right]$ (Pokhodylo, Shykka, Goreshnik et al., 2020). Conversely, in the triazoles unsubstituted at the 5-position, [1-(3-bromo- or 4-fluorophenyl)- $1 \mathrm{H}$ 1,2,3-triazol-4-yl]methyl methylphosphonate, these angle are 22.9 (3) and $15.7(2)^{\circ}$, respectively (Pokhodylo, Shyyka et al., 2019).

\section{Supramolecular features}

As shown in Fig. 2 and Table 1, the extended structure of the title compound features a number of directional intermolecular interactions. The molecules are linked by $\mathrm{O} 2-$ $\mathrm{H} 2 \cdots \mathrm{O} 1^{\mathrm{i}}$ and $\mathrm{C} 11-\mathrm{H} 11 \cdots \mathrm{N} 3^{\mathrm{ii}}$ (see Table 1 for symmetry codes) interactions into an infinite ribbon propagating in the [001] direction. The ribbons are interconnected by a weak $\mathrm{C} 5-\mathrm{H} 5 \cdots \mathrm{O} 2^{\mathrm{iii}}$ interaction into layers (Fig. 3).

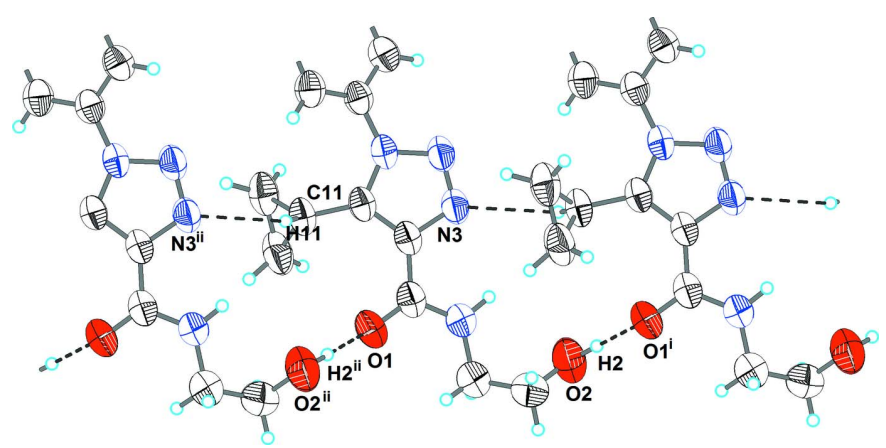

Figure 2

The hydrogen-bonded ribbon in the title compound. Hydrogen bonds are shown as dashed lines. The symmetry codes are as in Table 1 . 


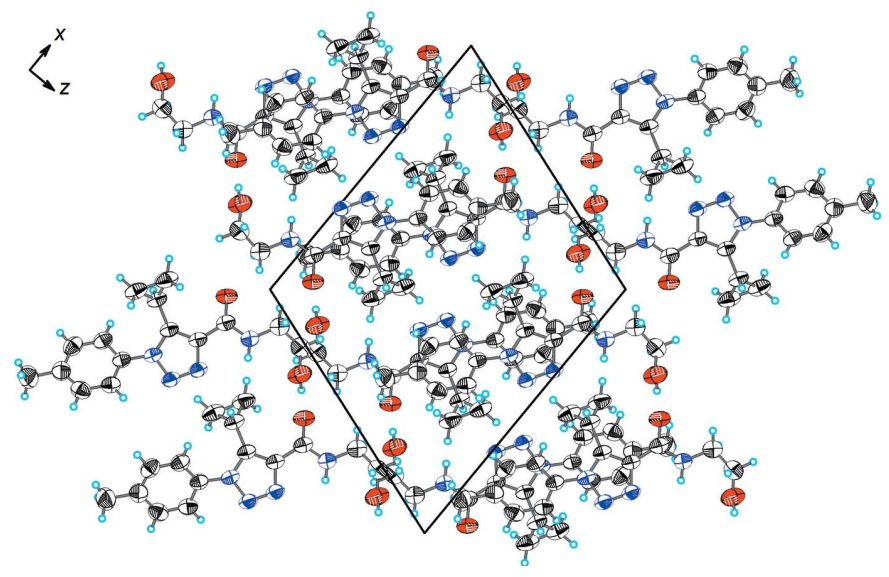

Figure 3

A view along the $b$-axis direction of the crystal packing of the title compound.

\section{Hirshfeld surface analysis}

The significant interactions among the molecules of the title compound can be visualized qualitatively through Hirshfeld surface analysis (Spackman \& Jayatilaka, 2009). The mapping of the normalized contact distance $\left(d_{\text {norm }}\right)$ was performed using the CrystalExplorer software (Turner et al., 2017). The most prominent interactions (short contact areas) are indicated on the Hirshfeld surfaces in red, whereas long contacts are shown in blue. Fingerprint plots were produced to show the intermolecular surface bond distances with the regions highlighted for $\mathrm{O} \cdots \mathrm{H} / \mathrm{H} \cdots \mathrm{O}$ and $\mathrm{N} \cdots \mathrm{H} / \mathrm{H} \cdots \mathrm{N}$ interactions (Fig. 4). The contributions to the surface area for such contacts are $12.9 \%$ and $15.4 \%$, respectively. The relatively low percentage of $\mathrm{C} \cdots \mathrm{H} / \mathrm{H} \cdots \mathrm{C}$ contacts $(13.2 \%)$ indicates the small contribution of $\mathrm{C}-\mathrm{H} \cdots \pi$ interactions for consolidating the crystal packing. The contribution to the surface area for $\mathrm{H} \cdot \mathrm{H}$ contacts is $55.5 \%$.

\section{Database survey}

The most closest related compounds containing a similar 1-aryl-1H-1,2,3-triazole-4-carboxamide skeleton to the title

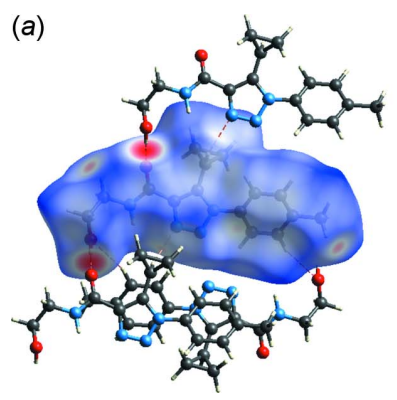

(b)

(c)
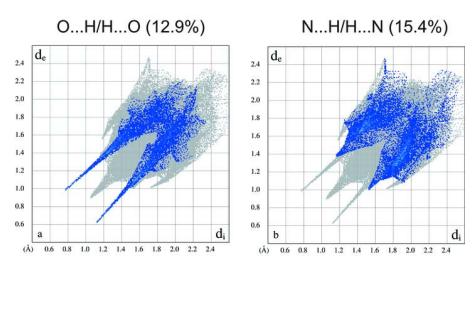

Figure 4

(a) Hirshfeld surface for the title compound mapped with $d_{\text {norm }}$ over the range -0.68 to 1.46 showing the $\mathrm{O}-\mathrm{H} \cdots \mathrm{O}, \mathrm{C}-\mathrm{H} \cdots \mathrm{N}$ and $\mathrm{C}-\mathrm{H} \cdots \mathrm{O}$ hydrogen-bonded contacts. Fingerprint plots resolved into $(b) \mathrm{O} \cdots \mathrm{H} /$ $\mathrm{H} \cdots \mathrm{O}$ and $(c) \mathrm{N} \cdots \mathrm{H} / \mathrm{H} \cdots \mathrm{N}$ contacts. Neighbouring molecules associated with close contacts are also shown. compound but with different substituents on the amide are: $\mathrm{N}$-(4-chlorophenyl)-5-cyclopropyl-1-(4-methoxyphenyl)- $1 \mathrm{H}$ 1,2,3-triazole-4-carboxamide (Pokhodylo \& Slyvka et al., 2020), (S)-1-(4-chlorophenyl)- $N$-(1-hydroxy-3-phenylpropan-2-yl)-5-methyl-1H-1,2,3-triazole-4-carboxamide [Cambridge Structural Database (Version 2021.1; Groom et al., 2016) refcode ZIPSEY; Shen et al., 2013], 1-(4-chlorophenyl)-5-methyl- $N$-[(3-phenyl-1,2-oxazol-5-yl)methyl]-1H1,2,3-triazole-4-carboxamide (II) (LELHOB; Niu et al., 2013), (5-methyl-1-[8-(trifluoromethyl)quinolin-4-yl]-1H-1,2,3-triazol-4-yl)morpholino)methanone (III) (LOHWIP; Anuradha et al., 2008) and 1-(3-amino-5-(3-hydroxy-3-methylbut-1-yn-1yl)phenyl)- $N$-butyl-1 $H$-1,2,3-triazole-4-carboxamide (IV) (BEBJEZ; Li et al., 2012).

Compounds (I) and (II) crystallize in the monoclinic crystal system with space groups $P 2_{1}$ and $P 2_{1} / \mathrm{c}$, respectively, while compounds (III) and (IV) crystallize in the triclinic space group $P \overline{1}$. Structure (I) contains two crystallographically independent molecules, the hydroxyl groups of which participate in intermolecular $\mathrm{O}-\mathrm{H} \cdots \mathrm{O}$ hydrogen bonds. In contrast to the molecular structure of title compound, the torsion angles between the phenyl rings and triazole rings in (I) are $-45.2(6)^{\circ}(\mathrm{C} 5-\mathrm{C} 6-\mathrm{N} 1-\mathrm{N} 2)$ and $39.9(6)^{\circ}\left(\mathrm{C1}^{\prime}-\right.$ $\left.\mathrm{C}^{\prime}-\mathrm{N} 1^{\prime}-\mathrm{N} 2^{\prime}\right)$; the analogous value in (II) is $19.2(2)^{\circ}$. In structure (II), the carboxamide groups connect neighbouring molecules into infinite chains by means of $\mathrm{N}-\mathrm{H} \cdots \mathrm{O}$ hydrogen bonds. The molecules in structures (III) and (IV) are connected by $\mathrm{N}-\mathrm{H} \cdots \mathrm{O}(\mathrm{oxazol})$ contacts. Similarly to (I) and (II), structure (III) contains a 5-methyl substituent at the triazole ring; as a result of the significant steric hindrance of 8-(trifluoromethyl)quinoline, the dihedral angle between the rings is $54.7^{\circ}$. The phenyl and triazole rings in (IV) are close to coplanar $\left(7.5^{\circ}\right)$, while the hydroxyl, carboxamide and amino groups participate in $\mathrm{O}-\mathrm{H} \cdots \mathrm{O}$ and $\mathrm{N}-\mathrm{H} \cdots \mathrm{O}$ hydrogen bonds. Finally, two copper(I) $\pi$-complexes of compositions $\left[\mathrm{Cu}\left(\mathrm{C}_{12} \mathrm{H}_{13} \mathrm{~N}_{5} \mathrm{O}\right)\left(\mathrm{NO}_{3}\right)\right] \cdot 0.5 \mathrm{H}_{2} \mathrm{O}$ and $\left[\mathrm{Cu}\left(\mathrm{C}_{12} \mathrm{H}_{13} \mathrm{~N}_{5} \mathrm{O}\right)\left(\mathrm{CF}_{3^{-}}\right.\right.$ COO) $]\left(\mathrm{C}_{12} \mathrm{H}_{13} \mathrm{~N}_{5} \mathrm{O}\right.$ is $\mathrm{N}$-allyl-5-amino-1-phenyl- $1 H$-1,2,3-triazole-4-carboxamide) were obtained by electrochemical synthesis (ZEQTOG and ZEQTUM; Slyvka et al., 2012). Crystals of these compounds are monoclinic, space group $C 2 / c$ : in both structures, the $N$-allyl- $1 H$-1,2,3-triazole-4carboxamide motif acts as a bridging chelating ligand and forms with the copper(I) atoms infinite chains containing $\left[\mathrm{CuC}_{4} \mathrm{NO}\right]$ seven-membered rings.

\section{Synthesis and crystallization}

5-Cyclopropyl-1-p-tolyl-1H-1,2,3-triazole-4-carboxylic acid (Pokhodylo et al., 2017) (1.22 g, $5.00 \mathrm{mmol})$ was added to a solution of 1,1'-carbonyldiimidazole (CDI, $0.81 \mathrm{~g}, 5.0 \mathrm{mmol}$ ) in dry acetonitrile $(5 \mathrm{ml})$ and the mixture was kept for $30 \mathrm{~min}$ at $323 \mathrm{~K}$. Then, $0.3 \mathrm{ml}$ of 2-aminoethanol $(0.31 \mathrm{~g}, 5.00 \mathrm{mmol})$ was added, and the mixture was heated at $343 \mathrm{~K}$ for $1 \mathrm{~h}$. After cooling to room temperature, water $(30 \mathrm{ml})$ was added. The precipitate was filtered off, washed with water on a filter, crystallized from diluted ethanol solution, and dried in air to give the title compound as colourless crystals, m.p. 396-397 K. 


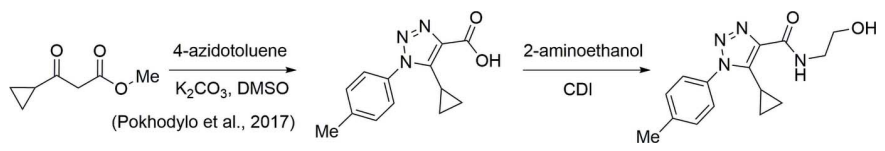

Figure 5

Synthesis of 5-cyclopropyl- $N$-(2-hydroxyethyl)-1-(p-tolyl)-1H-1,2,3-triazole-4-carboxamide.

The reaction scheme is shown in Fig. 5. IR $\left(\mathrm{KBr}, v, \mathrm{~cm}^{-1}\right)$ : $1685(\mathrm{C}=\mathrm{O}) ; 3370(\mathrm{~N}-\mathrm{H}) .{ }^{1} \mathrm{H}$ NMR: $\left(400 \mathrm{MHz}, \mathrm{DMSO}-d_{6}\right)$ : $\delta=0.85-0.91\left(m, 2 \mathrm{H}, \mathrm{CH}_{2}\right), 0.98-1.02\left(m, 2 \mathrm{H}, \mathrm{CH}_{2}\right), 1.95-1.99$ $(m, 1 \mathrm{H}, \mathrm{CH}), 2.46\left(c, 3 \mathrm{H}, \mathrm{CH}_{3}\right), 3.37\left(q, J=5.8 \mathrm{~Hz}, 2 \mathrm{H}, \mathrm{CH}_{2} \mathrm{~N}\right)$, $3.54\left(q, J=5.8 \mathrm{~Hz}, 2 \mathrm{H}, \mathrm{CH}_{2} \mathrm{O}\right), 4.58(t, J=6.0, \mathrm{~Hz}, 1 \mathrm{H}, \mathrm{OH})$, $7.37\left(d, J=7.6 \mathrm{~Hz}, 2 \mathrm{H}, \mathrm{H}_{\mathrm{Ar}^{-}}-3,5\right), 7.43\left(d, J=7.6 \mathrm{~Hz}, 2 \mathrm{H}, \mathrm{H}_{\mathrm{Ar}^{-}}\right.$ 2,6), $8.14(t, J=5.4 \mathrm{~Hz}, 1 \mathrm{H}, \mathrm{NH}) .{ }^{13} \mathrm{C}$ NMR: $(101 \mathrm{MHz}$, DMSO- $\left.d_{6}\right): \delta=5.3(\mathrm{CH}), 8.2\left(2 \times \mathrm{CH}_{2}\right), 21.1\left(\mathrm{CH}_{3}\right), 42.3$ $\left(\mathrm{CH}_{2} \mathrm{~N}\right), 59.5\left(\mathrm{CH}_{2} \mathrm{O}\right), 126.5\left(2 \times \mathrm{CH}_{\mathrm{Ar}^{-}}-2,6\right), 130.1\left(2 \times \mathrm{CH}_{\mathrm{Ar}^{-}}\right.$ 3,5), $133.7\left(\mathrm{C}_{\mathrm{Ar}}-1\right), 137.2\left(\mathrm{C}_{\text {Triazole }}-4\right), 139.2\left(\mathrm{C}_{\mathrm{Ar}^{-4}}\right), 144.6$ $\left(\mathrm{C}_{\text {Triazole }}-5\right), 161.8(\mathrm{C}=\mathrm{O})$. MS, $m / z=287\left(M^{+}+1\right)$. Calculated for $\mathrm{C}_{15} \mathrm{H}_{18} \mathrm{~N}_{4} \mathrm{O}_{2},(\%)$ : $\mathrm{C} 62.92 ; \mathrm{H} 6.34, \mathrm{~N}$ 19.57. Found (\%): C $62.83 ; \mathrm{H} 6.57, \mathrm{~N} 19.32$.

\section{Refinement}

Crystal data, data collection and structure refinement details are summarized in Table 2. N-bound and $\mathrm{O}$-bound $\mathrm{H}$ atoms were located in difference-Fourier maps and refined isotropically. C-bound $\mathrm{H}$ atoms were positioned geometrically and refined using a riding model, with $\mathrm{C}-\mathrm{H}=0.93-0.98 \AA$ and $U_{\text {iso }}(\mathrm{H})=1.2 U_{\text {eq }}(\mathrm{C})$ or $1.5 U_{\text {eq }}(\mathrm{C}$-methyl $)$.

\section{Funding information}

The authors are grateful to the Ministry of Education and Science of Ukraine for financial support of this project (grant No. 0121U107777).

\section{References}

Anuradha, N., Thiruvalluvar, A., Mahalinga, M. \& Butcher, R. J. (2008). Acta Cryst. E64, o2375.

Bekheit, M. S., Mohamed, H. A., Abdel-Wahab, B. F. \& Fouad, M. A. (2021). Med. Chem. Res. 30, 1125-1138.

Dolomanov, O. V., Bourhis, L. J., Gildea, R. J., Howard, J. A. K. \& Puschmann, H. (2009). J. Appl. Cryst. 42, 339-341.

Elamari, H., Slimi, R., Chabot, G. G., Quentin, L., Scherman, D. \& Girard, C. (2013). Eur. J. Med. Chem. 60, 360-364.

Giannini, G. \& Battistuzzi, G. (2015). Bioorg. Med. Chem. Lett. 25, 462-465.

Groom, C. R., Bruno, I. J., Lightfoot, M. P. \& Ward, S. C. (2016). Acta Cryst. B72, 171-179.

Jadhav, R. P., Raundal, H. N., Patil, A. A. \& Bobade, V. D. (2017). J. Saudi Chem. Soc. 21, 152-159.

Krajczyk, A., Kulinska, K., Kulinski, T., Hurst, B. L., Day, C. W., Smee, D. F., Ostrowski, T., Januszczyk, P. \& Zeidler, J. (2014). Antivir. Chem. Chemother. 23, 161-171.

Li, Y.-J., Xu, L., Yang, W.-L., Liu, H.-B., Lai, S.-W., Che, C.-M. \& Li, Y.-L. (2012). Chem. Eur. J. 18, 4782-4790.

Niu, T.-F., Lv, M.-F., Wang, L., Yi, W.-B. \& Cai, C. (2013). Org. Biomol. Chem. 11, 1040-1048.
Table 2

Experimental details.

\begin{tabular}{|c|c|}
\hline \multicolumn{2}{|l|}{ Crystal data } \\
\hline Chemical formula & $\mathrm{C}_{15} \mathrm{H}_{18} \mathrm{~N}_{4} \mathrm{O}_{2}$ \\
\hline$M_{\mathrm{r}}$ & 286.33 \\
\hline Crystal system, space group & Monoclinic, $P 2_{1} / c$ \\
\hline Temperature $(\mathrm{K})$ & 293 \\
\hline$a, b, c(\AA)$ & $14.3158(6), 8.3972(3), 13.0871(4)$ \\
\hline$\beta\left(^{\circ}\right)$ & $108.040(4)$ \\
\hline$V\left(\AA^{3}\right)$ & $1495.90(10)$ \\
\hline$Z$ & 4 \\
\hline Radiation type & Мо $K \alpha$ \\
\hline$\mu\left(\mathrm{mm}^{-1}\right)$ & 0.09 \\
\hline Crystal size $(\mathrm{mm})$ & $0.5 \times 0.4 \times 0.06$ \\
\hline \multicolumn{2}{|l|}{ Data collection } \\
\hline Diffractometer & Oxford Diffraction Xcalibur3 CCD \\
\hline Absorption correction & $\begin{array}{l}\text { Multi-scan (CrysAlis RED; Oxford } \\
\text { Diffraction, 2004) }\end{array}$ \\
\hline$T_{\min }, T_{\max }$ & $0.935,0.988$ \\
\hline $\begin{array}{l}\text { No. of measured, independent and } \\
\text { observed }[I>2 \sigma(I)] \text { reflections }\end{array}$ & $8400,2632,1621$ \\
\hline$R_{\text {int }}$ & 0.032 \\
\hline$(\sin \theta / \lambda)_{\max }\left(\AA^{-1}\right)$ & 0.595 \\
\hline \multicolumn{2}{|l|}{ Refinement } \\
\hline$R\left[F^{2}>2 \sigma\left(F^{2}\right)\right], w R\left(F^{2}\right), S$ & $0.047,0.099,1.05$ \\
\hline No. of reflections & 2632 \\
\hline No. of parameters & 199 \\
\hline $\mathrm{H}$-atom treatment & $\begin{array}{l}\mathrm{H} \text { atoms treated by a mixture of } \\
\text { independent and constrained } \\
\text { refinement }\end{array}$ \\
\hline$\Delta \rho_{\max }, \Delta \rho_{\min }\left(\mathrm{e} \AA^{-3}\right)$ & $0.14,-0.20$ \\
\hline
\end{tabular}

Computer programs: CrysAlis CCD and CrysAlis RED (Oxford Diffraction, 2004), SHELXT (Sheldrick, 2015a), SHELXL (Sheldrick, 2015b) and OLEX2 (Dolomanov et al., 2009).

Obianom, O. N., Ai, Y., Li, Y., Yang, W., Guo, D., Yang, H., Sakamuru, S., Xia, M., Xue, F. \& Shu, Y. (2019). J. Med. Chem. 62, 727741.

Oxford Diffraction (2004). CrysAlis CCD and CrysAlis RED. Oxford Diffraction, Yarnton, England.

Pokhodylo, N., Manko, N., Finiuk, N., Klyuchivska, O., Matiychuk, V., Obushak, M. \& Stoika, R. (2021). J. Mol. Struct. 2021 Art. 131146.

Pokhodylo, N., Shyyka, O., Finiuk, N. \& Stoika, R. (2020). Ukr. Biochem. J. 92, 23-32.

Pokhodylo, N., Slyvka, Y. \& Pavlyuk, V. (2020). Acta Cryst. E76, 756760.

Pokhodylo, N. T. \& Obushak, M. D. (2019). Russ. J. Org. Chem. 55, 1241-1243.

Pokhodylo, N. T., Shyyka, O. Ya., Goreshnik, E. A. \& Obushak, M. D. (2020). ChemistrySelect, 5, 260-264.

Pokhodylo, N. T., Shyyka, O. Ya., Matiychuk, V. S., Obushak, M. D. \& Pavlyuk, V. V. (2017). ChemistrySelect, 2, 5871-5876.

Pokhodylo, N. T., Shyyka, O. Ya., Tupychak, M. A., Slyvka, Yu. I. \& Obushak, M. D. (2019). Chem. Heterocycl. Compd, 55, 374378.

Prasad, B., Lakshma Nayak, V., Srikanth, P. S., Baig, M. F., Subba Reddy, N. V., Babu, K. S. \& Kamal, A. (2019). Bioorg. Chem. 83, 535-548.

Reddy, V. G., Bonam, S. R., Reddy, T. S., Akunuri, R., Naidu, V. G. M., Nayak, V. L., Bhargava, S. K., Kumar, H. S., Srihari, P. \& Kamal, A. (2018). Eur. J. Med. Chem. 144, 595-611.

Sheldrick, G. M. (2015a). Acta Cryst. A71, 3-8.

Sheldrick, G. M. (2015b). Acta Cryst. C71, 3-8.

Shen, G.-L., Chen, Z.-B., Wu, Z.-F. \& Dong, H.-S. (2013). J. Heterocycl. Chem. 50, 781-786.

Shyyka, O. Ya., Pokhodylo, N. T. \& Finiuk, N. S. (2019). Biopolym. Cell, 35, 321-330. 
Slyvka, Yu. I., Pavlyuk, A. V., Ardan, B. R., Pokhodilo, N. T., Goreshnik, E. A. \& Demchenko, P. Yu. (2012). Russ. J. Inorg. Chem. 57, 815-821.

Spackman, M. A. \& Jayatilaka, D. (2009). CrystEngComm, 11, 1932.

Taddei, M., Ferrini, S., Giannotti, L., Corsi, M., Manetti, F., Giannini, G., Vesci, L., Milazzo, F. M., Alloatti, D., Guglielmi, M. B., Castorina, M., Cervoni, M. L., Barbarino, M., Foderà, R., Carollo, V., Pisano, C., Armaroli, S. \& Cabri, W. (2014). J. Med. Chem. 57, 2258-2274.
Turner, M. J., Mckinnon, J. J., Wolff, S. K., Grimwood, D. J., Spackman, P. R., Jayatilaka, D. \& Spackman, M. A. (2017). CrystalExplorer17. The University of Western Australia. http:// hirshfeldsurface.net

Wang, L., Xu, S., Liu, X., Chen, X., Xiong, H., Hou, S., Zou, W., Tang, Q., Zheng, P. \& Zhu, W. (2018). Bioorg. Chem. 77, 370-380.

Wang, Z., Gao, Y., Hou, Y., Zhang, C., Yu, S. J., Bian, Q., Li, Z. M. \& Zhao, W. G. (2014). Eur. J. Med. Chem. 86, 87-94.

Zhou, S., Liao, H., Liu, M., Feng, G., Fu, B., Li, R., Cheng, M., Zhao, Y. \& Gong, P. (2014). Bioorg. Med. Chem. 22, 6438-6452. 


\section{supporting information}

Acta Cryst. (2021). E77, 1043-1047 [https://doi.org/10.1107/S2056989021009774]

Synthesis, crystal structure and Hirshfeld surface analysis of 5-cyclopropyl-

N-(2-hydroxyethyl)-1-(4-methylphenyl)-1H-1,2,3-triazole-4-carboxamide

Nazariy T. Pokhodylo, Yurii Slyvka and Volodymyr Pavlyuk

Computing details

Data collection: CrysAlis CCD (Oxford Diffraction, 2004); cell refinement: CrysAlis CCD (Oxford Diffraction, 2004); data reduction: CrysAlis RED (Oxford Diffraction, 2004); program(s) used to solve structure: ShelXT (Sheldrick, 2015a); program(s) used to refine structure: SHELXL (Sheldrick, 2015b); molecular graphics: OLEX2 (Dolomanov et al., 2009); software used to prepare material for publication: OLEX2 (Dolomanov et al., 2009).

5-Cyclopropyl-N-(2-hydroxyethyl)-1-(4-methylphenyl)-1H-1,2,3-triazole-4-carboxamide

\section{Crystal data}

$\mathrm{C}_{15} \mathrm{H}_{18} \mathrm{~N}_{4} \mathrm{O}_{2}$

$M_{r}=286.33$

Monoclinic, $P 2_{1} / c$

$a=14.3158(6) \AA$

$b=8.3972(3) \AA$

$c=13.0871(4) \AA$

$\beta=108.040(4)^{\circ}$

$V=1495.90(10) \AA^{3}$

$Z=4$

Data collection

Oxford Diffraction Xcalibur3 CCD diffractometer

$\omega$ scans

Absorption correction: multi-scan

(CrysAlis RED; Oxford Diffraction, 2004)

$T_{\text {min }}=0.935, T_{\text {max }}=0.988$

8400 measured reflections

\section{Refinement}

Refinement on $F^{2}$

Least-squares matrix: full

$R\left[F^{2}>2 \sigma\left(F^{2}\right)\right]=0.047$

$w R\left(F^{2}\right)=0.099$

$S=1.05$

2632 reflections

199 parameters

0 restraints

Primary atom site location: dual
$F(000)=608$

$D_{\mathrm{x}}=1.271 \mathrm{Mg} \mathrm{m}^{-3}$

Mo $K \alpha$ radiation, $\lambda=0.71073 \AA$

Cell parameters from 1679 reflections

$\theta=2.9-26.4^{\circ}$

$\mu=0.09 \mathrm{~mm}^{-1}$

$T=293 \mathrm{~K}$

Lamina, clear colourless

$0.5 \times 0.4 \times 0.06 \mathrm{~mm}$

2632 independent reflections

1621 reflections with $I>2 \sigma(I)$

$R_{\text {int }}=0.032$

$\theta_{\text {max }}=25.0^{\circ}, \theta_{\min }=2.9^{\circ}$

$h=-17 \rightarrow 17$

$k=-9 \rightarrow 9$

$l=-15 \rightarrow 8$

Hydrogen site location: mixed

$\mathrm{H}$ atoms treated by a mixture of independent and constrained refinement

$w=1 /\left[\sigma^{2}\left(F_{\mathrm{o}}^{2}\right)+(0.035 P)^{2}+0.180 P\right]$ where $P=\left(F_{\mathrm{o}}^{2}+2 F_{\mathrm{c}}^{2}\right) / 3$

$(\Delta / \sigma)_{\max }<0.001$

$\Delta \rho_{\max }=0.14 \mathrm{e} \AA^{-3}$

$\Delta \rho_{\min }=-0.20 \mathrm{e}^{-3}$ 


\section{Special details}

Geometry. All esds (except the esd in the dihedral angle between two 1.s. planes) are estimated using the full covariance matrix. The cell esds are taken into account individually in the estimation of esds in distances, angles and torsion angles; correlations between esds in cell parameters are only used when they are defined by crystal symmetry. An approximate (isotropic) treatment of cell esds is used for estimating esds involving l.s. planes.

Refinement. 1. Fixed Uiso At 1.2 times of: All $\mathrm{C}(\mathrm{H})$ groups, All $\mathrm{C}(\mathrm{H}, \mathrm{H})$ groups At 1.5 times of: All $\mathrm{C}(\mathrm{H}, \mathrm{H}, \mathrm{H})$ groups 2.a Ternary $\mathrm{CH}$ refined with riding coordinates: $\mathrm{C} 11(\mathrm{H} 11)$ 2.b Secondary $\mathrm{CH} 2$ refined with riding coordinates: C12(H12A,H12B), C13(H13A,H13B), C14(H14A,H14B), C15(H15A,H15B) 2.c Aromatic/amide H refined with riding coordinates: $\mathrm{C} 2(\mathrm{H} 2 \mathrm{~A}), \mathrm{C} 3(\mathrm{H} 3), \mathrm{C} 5(\mathrm{H} 5), \mathrm{C} 6(\mathrm{H} 6)$ 2.d Idealised Me refined as rotating group: C7(H7A,H7B,H7C)

Fractional atomic coordinates and isotropic or equivalent isotropic displacement parameters $\left(\AA^{2}\right)$

\begin{tabular}{|c|c|c|c|c|}
\hline & $x$ & $y$ & $z$ & $U_{\text {iso }} * / U_{\text {eq }}$ \\
\hline $\mathrm{O} 1$ & $0.13456(11)$ & $0.3294(2)$ & $0.60427(12)$ & $0.0974(5)$ \\
\hline $\mathrm{O} 2$ & $0.06831(14)$ & $0.3928(2)$ & $0.21808(14)$ & $0.1042(6)$ \\
\hline $\mathrm{H} 2$ & $0.092(2)$ & $0.318(4)$ & $0.177(3)$ & $0.171(14)^{*}$ \\
\hline N1 & $0.44653(12)$ & $0.35154(18)$ & $0.67420(11)$ & $0.0570(4)$ \\
\hline $\mathrm{N} 2$ & $0.43955(13)$ & $0.3492(2)$ & $0.56776(12)$ & $0.0687(5)$ \\
\hline N3 & $0.34622(13)$ & $0.3512(2)$ & $0.51377(12)$ & $0.0680(5)$ \\
\hline N4 & $0.14491(15)$ & $0.3789(3)$ & $0.44072(15)$ & $0.0846(6)$ \\
\hline $\mathrm{H} 4$ & $0.1840(16)$ & $0.397(3)$ & $0.4005(17)$ & $0.090(7)^{*}$ \\
\hline $\mathrm{C} 1$ & $0.54283(14)$ & $0.3518(2)$ & $0.75104(14)$ & $0.0573(5)$ \\
\hline $\mathrm{C} 2$ & $0.56125(17)$ & $0.2755(2)$ & $0.84840(16)$ & $0.0660(6)$ \\
\hline $\mathrm{H} 2 \mathrm{~A}$ & 0.510965 & 0.223701 & 0.866145 & $0.079^{*}$ \\
\hline $\mathrm{C} 3$ & 0.65554 (19) & $0.2771(3)$ & $0.91933(17)$ & $0.0755(6)$ \\
\hline H3 & 0.667851 & 0.226965 & 0.985570 & $0.091^{*}$ \\
\hline $\mathrm{C} 4$ & $0.73224(17)$ & $0.3510(3)$ & $0.89461(18)$ & $0.0754(6)$ \\
\hline $\mathrm{C} 5$ & $0.71121(17)$ & $0.4244(3)$ & 0.79535 (19) & $0.0768(6)$ \\
\hline H5 & 0.761557 & 0.474207 & 0.776551 & $0.092 *$ \\
\hline C6 & $0.61784(16)$ & $0.4254(2)$ & $0.72389(17)$ & $0.0658(5)$ \\
\hline H6 & 0.605361 & 0.475465 & 0.657611 & $0.079 *$ \\
\hline $\mathrm{C} 7$ & $0.83533(18)$ & $0.3513(4)$ & $0.9729(2)$ & $0.1156(10)$ \\
\hline $\mathrm{H} 7 \mathrm{~A}$ & 0.853395 & 0.457928 & 0.997559 & $0.173^{*}$ \\
\hline H7B & 0.880342 & 0.311663 & 0.937725 & $0.173^{*}$ \\
\hline $\mathrm{H} 7 \mathrm{C}$ & 0.837630 & 0.284323 & 1.033110 & $0.173^{*}$ \\
\hline $\mathrm{C} 8$ & $0.35570(14)$ & $0.3551(2)$ & $0.68719(14)$ & $0.0546(5)$ \\
\hline C9 & $0.29293(14)$ & $0.3532(2)$ & $0.58406(14)$ & $0.0583(5)$ \\
\hline $\mathrm{C} 10$ & $0.18463(16)$ & $0.3519(2)$ & $0.54485(16)$ & $0.0671(5)$ \\
\hline C11 & $0.33673(14)$ & $0.3634(2)$ & $0.79130(14)$ & $0.0616(5)$ \\
\hline H11 & 0.329955 & 0.260032 & 0.823068 & $0.074 *$ \\
\hline $\mathrm{C} 12$ & $0.27363(19)$ & $0.4924(3)$ & $0.81202(17)$ & $0.0882(7)$ \\
\hline $\mathrm{H} 12 \mathrm{~A}$ & 0.249069 & 0.572020 & 0.756496 & $0.106^{*}$ \\
\hline H12B & 0.228996 & 0.465129 & 0.851979 & $0.106^{*}$ \\
\hline $\mathrm{C} 13$ & $0.37951(19)$ & $0.4924(3)$ & $0.87037(17)$ & $0.0791(7)$ \\
\hline H13A & 0.399859 & 0.464995 & 0.946092 & $0.095 *$ \\
\hline H13B & 0.419936 & 0.571906 & 0.850592 & $0.095^{*}$ \\
\hline $\mathrm{C} 14$ & $0.03984(18)$ & $0.3804(3)$ & $0.38523(18)$ & $0.0979(8)$ \\
\hline H14A & 0.016031 & 0.489258 & 0.378087 & $0.117^{*}$ \\
\hline
\end{tabular}


supporting information

\begin{tabular}{lllll} 
H14B & 0.005972 & 0.321759 & 0.427025 & $0.117^{*}$ \\
C15 & $0.01844(17)$ & $0.3080(3)$ & $0.27817(18)$ & $0.0946(8)$ \\
H15A & 0.039484 & 0.197635 & 0.285111 & $0.114^{*}$ \\
H15B & -0.051722 & 0.310769 & 0.241585 & $0.114^{*}$ \\
\hline
\end{tabular}

Atomic displacement parameters $\left(\AA^{2}\right)$

\begin{tabular}{lllllll}
\hline & $U^{11}$ & $U^{22}$ & $U^{33}$ & $U^{12}$ & $U^{13}$ & $U^{23}$ \\
\hline O1 & $0.0884(11)$ & $0.1413(16)$ & $0.0775(10)$ & $-0.0021(10)$ & $0.0476(9)$ & $0.0143(9)$ \\
O2 & $0.1267(15)$ & $0.1162(15)$ & $0.0851(12)$ & $-0.0073(11)$ & $0.0552(11)$ & $-0.0083(10)$ \\
N1 & $0.0729(11)$ & $0.0568(10)$ & $0.0487(9)$ & $-0.0034(8)$ & $0.0296(8)$ & $-0.0040(8)$ \\
N2 & $0.0769(13)$ & $0.0840(13)$ & $0.0532(10)$ & $-0.0042(10)$ & $0.0317(9)$ & $-0.0090(9)$ \\
N3 & $0.0760(13)$ & $0.0828(13)$ & $0.0525(9)$ & $-0.0047(10)$ & $0.0302(9)$ & $-0.0081(8)$ \\
N4 & $0.0674(13)$ & $0.1313(18)$ & $0.0594(12)$ & $-0.0073(11)$ & $0.0258(10)$ & $0.0050(11)$ \\
C1 & $0.0700(14)$ & $0.0492(12)$ & $0.0575(12)$ & $0.0002(10)$ & $0.0271(11)$ & $-0.0064(10)$ \\
C2 & $0.0834(17)$ & $0.0572(13)$ & $0.0609(13)$ & $-0.0025(11)$ & $0.0273(12)$ & $-0.0031(10)$ \\
C3 & $0.0962(19)$ & $0.0669(15)$ & $0.0621(13)$ & $0.0136(13)$ & $0.0228(14)$ & $-0.0030(11)$ \\
C4 & $0.0750(16)$ & $0.0746(15)$ & $0.0752(15)$ & $0.0140(13)$ & $0.0209(12)$ & $-0.0165(13)$ \\
C5 & $0.0738(17)$ & $0.0738(16)$ & $0.0895(17)$ & $0.0014(12)$ & $0.0349(14)$ & $-0.0096(13)$ \\
C6 & $0.0758(15)$ & $0.0599(13)$ & $0.0684(13)$ & $0.0026(11)$ & $0.0322(12)$ & $0.0003(10)$ \\
C7 & $0.0830(19)$ & $0.155(3)$ & $0.1007(19)$ & $0.0267(17)$ & $0.0160(15)$ & $-0.0231(18)$ \\
C8 & $0.0729(13)$ & $0.0448(11)$ & $0.0535(11)$ & $-0.0021(10)$ & $0.0304(10)$ & $-0.0007(9)$ \\
C9 & $0.0719(14)$ & $0.0575(13)$ & $0.0538(12)$ & $-0.0048(10)$ & $0.0315(10)$ & $-0.0014(10)$ \\
C10 & $0.0796(15)$ & $0.0702(14)$ & $0.0591(13)$ & $-0.0042(12)$ & $0.0328(12)$ & $-0.0001(11)$ \\
C11 & $0.0893(15)$ & $0.0516(12)$ & $0.0536(11)$ & $-0.0055(11)$ & $0.0364(10)$ & $-0.0003(10)$ \\
C12 & $0.116(2)$ & $0.0920(18)$ & $0.0739(15)$ & $0.0236(15)$ & $0.0541(15)$ & $0.0002(12)$ \\
C13 & $0.116(2)$ & $0.0663(15)$ & $0.0657(13)$ & $-0.0099(13)$ & $0.0447(14)$ & $-0.0104(11)$ \\
C14 & $0.0787(18)$ & $0.147(2)$ & $0.0738(15)$ & $0.0057(15)$ & $0.0315(13)$ & $-0.0002(15)$ \\
C15 & $0.0722(16)$ & $0.125(2)$ & $0.0884(18)$ & $-0.0097(14)$ & $0.0268(14)$ & $-0.0072(15)$ \\
& & & & & & \\
\hline
\end{tabular}

Geometric parameters $\left(\AA,{ }^{\circ}\right)$

\begin{tabular}{llll}
\hline $\mathrm{O} 1-\mathrm{C} 10$ & $1.224(2)$ & $\mathrm{C} 6-\mathrm{H} 6$ & 0.9300 \\
$\mathrm{O} 2-\mathrm{H} 2$ & $0.95(3)$ & $\mathrm{C} 7-\mathrm{H} 7 \mathrm{~A}$ & 0.9600 \\
$\mathrm{O} 2-\mathrm{C} 15$ & $1.408(3)$ & $\mathrm{C} 7-\mathrm{H} 7 \mathrm{~B}$ & 0.9600 \\
$\mathrm{~N} 1-\mathrm{N} 2$ & $1.3655(19)$ & $\mathrm{C} 7-\mathrm{H} 7 \mathrm{C}$ & 0.9600 \\
$\mathrm{~N} 1-\mathrm{C} 1$ & $1.433(2)$ & $\mathrm{C} 8-\mathrm{C} 9$ & $1.370(2)$ \\
$\mathrm{N} 1-\mathrm{C} 8$ & $1.363(2)$ & $\mathrm{C} 8-\mathrm{C} 11$ & $1.471(2)$ \\
$\mathrm{N} 2-\mathrm{N} 3$ & $1.304(2)$ & $\mathrm{C} 9-\mathrm{C} 10$ & $1.475(3)$ \\
$\mathrm{N} 3-\mathrm{C} 9$ & $1.365(2)$ & $\mathrm{C} 11-\mathrm{H} 11$ & 0.9800 \\
$\mathrm{~N} 4-\mathrm{H} 4$ & $0.89(2)$ & $\mathrm{C} 11-\mathrm{C} 12$ & $1.488(3)$ \\
$\mathrm{N} 4-\mathrm{C} 10$ & $1.324(3)$ & $\mathrm{C} 11-\mathrm{C} 13$ & $1.492(3)$ \\
$\mathrm{N} 4-\mathrm{C} 14$ & $1.454(3)$ & $\mathrm{C} 12-\mathrm{H} 12 \mathrm{~A}$ & 0.9700 \\
$\mathrm{C} 1-\mathrm{C} 2$ & $1.377(3)$ & $\mathrm{C} 12-\mathrm{H} 12 \mathrm{~B}$ & 0.9700 \\
$\mathrm{C} 1-\mathrm{C} 6$ & $1.378(3)$ & $\mathrm{C} 12-\mathrm{C} 13$ & $1.471(3)$ \\
$\mathrm{C} 2-\mathrm{H} 2 \mathrm{~A}$ & 0.9300 & $\mathrm{C} 13-\mathrm{H} 13 \mathrm{~A}$ & 0.9700 \\
$\mathrm{C} 2-\mathrm{C} 3$ & $1.381(3)$ & $\mathrm{C} 13-\mathrm{H} 13 \mathrm{~B}$ & 0.9700 \\
$\mathrm{C} 3-\mathrm{H} 3$ & 0.9300 & $\mathrm{C} 14-\mathrm{H} 14 \mathrm{~A}$ & 0.9700
\end{tabular}




\begin{tabular}{|c|c|c|c|}
\hline $\mathrm{C} 3-\mathrm{C} 4$ & $1.384(3)$ & $\mathrm{C} 14-\mathrm{H} 14 \mathrm{~B}$ & 0.9700 \\
\hline $\mathrm{C} 4-\mathrm{C} 5$ & $1.384(3)$ & $\mathrm{C} 14-\mathrm{C} 15$ & $1.470(3)$ \\
\hline $\mathrm{C} 4-\mathrm{C} 7$ & $1.514(3)$ & $\mathrm{C} 15-\mathrm{H} 15 \mathrm{~A}$ & 0.9700 \\
\hline $\mathrm{C} 5-\mathrm{H} 5$ & 0.9300 & $\mathrm{C} 15-\mathrm{H} 15 \mathrm{~B}$ & 0.9700 \\
\hline $\mathrm{C} 5-\mathrm{C} 6$ & $1.374(3)$ & & \\
\hline $\mathrm{C} 15-\mathrm{O} 2-\mathrm{H} 2$ & $108(2)$ & $\mathrm{N} 3-\mathrm{C} 9-\mathrm{C} 8$ & $109.33(17)$ \\
\hline $\mathrm{N} 2-\mathrm{N} 1-\mathrm{C} 1$ & $117.80(15)$ & $\mathrm{N} 3-\mathrm{C} 9-\mathrm{C} 10$ & $120.82(17)$ \\
\hline $\mathrm{C} 8-\mathrm{N} 1-\mathrm{N} 2$ & $110.87(15)$ & $\mathrm{C} 8-\mathrm{C} 9-\mathrm{C} 10$ & $129.85(16)$ \\
\hline $\mathrm{C} 8-\mathrm{N} 1-\mathrm{C} 1$ & $131.32(15)$ & $\mathrm{O} 1-\mathrm{C} 10-\mathrm{N} 4$ & $122.1(2)$ \\
\hline $\mathrm{N} 3-\mathrm{N} 2-\mathrm{N} 1$ & $106.95(14)$ & $\mathrm{O} 1-\mathrm{C} 10-\mathrm{C} 9$ & $122.64(19)$ \\
\hline $\mathrm{N} 2-\mathrm{N} 3-\mathrm{C} 9$ & $109.13(15)$ & $\mathrm{N} 4-\mathrm{C} 10-\mathrm{C} 9$ & $115.29(17)$ \\
\hline $\mathrm{C} 10-\mathrm{N} 4-\mathrm{H} 4$ & $119.3(14)$ & $\mathrm{C} 8-\mathrm{C} 11-\mathrm{H} 11$ & 115.0 \\
\hline $\mathrm{C} 10-\mathrm{N} 4-\mathrm{C} 14$ & $124.32(19)$ & $\mathrm{C} 8-\mathrm{C} 11-\mathrm{C} 12$ & $119.99(17)$ \\
\hline $\mathrm{C} 14-\mathrm{N} 4-\mathrm{H} 4$ & $116.4(14)$ & $\mathrm{C} 8-\mathrm{C} 11-\mathrm{C} 13$ & $121.51(16)$ \\
\hline $\mathrm{C} 2-\mathrm{C} 1-\mathrm{N} 1$ & $121.04(18)$ & $\mathrm{C} 12-\mathrm{C} 11-\mathrm{H} 11$ & 115.0 \\
\hline $\mathrm{C} 2-\mathrm{C} 1-\mathrm{C} 6$ & $120.41(19)$ & $\mathrm{C} 12-\mathrm{C} 11-\mathrm{C} 13$ & $59.16(14)$ \\
\hline $\mathrm{C} 6-\mathrm{C} 1-\mathrm{N} 1$ & $118.51(17)$ & $\mathrm{C} 13-\mathrm{C} 11-\mathrm{H} 11$ & 115.0 \\
\hline $\mathrm{C} 1-\mathrm{C} 2-\mathrm{H} 2 \mathrm{~A}$ & 120.5 & $\mathrm{C} 11-\mathrm{C} 12-\mathrm{H} 12 \mathrm{~A}$ & 117.7 \\
\hline $\mathrm{C} 1-\mathrm{C} 2-\mathrm{C} 3$ & $119.0(2)$ & $\mathrm{C} 11-\mathrm{C} 12-\mathrm{H} 12 \mathrm{~B}$ & 117.7 \\
\hline $\mathrm{C} 3-\mathrm{C} 2-\mathrm{H} 2 \mathrm{~A}$ & 120.5 & $\mathrm{H} 12 \mathrm{~A}-\mathrm{C} 12-\mathrm{H} 12 \mathrm{~B}$ & 114.8 \\
\hline $\mathrm{C} 2-\mathrm{C} 3-\mathrm{H} 3$ & 119.1 & $\mathrm{C} 13-\mathrm{C} 12-\mathrm{C} 11$ & $60.56(14)$ \\
\hline $\mathrm{C} 2-\mathrm{C} 3-\mathrm{C} 4$ & $121.9(2)$ & $\mathrm{C} 13-\mathrm{C} 12-\mathrm{H} 12 \mathrm{~A}$ & 117.7 \\
\hline $\mathrm{C} 4-\mathrm{C} 3-\mathrm{H} 3$ & 119.1 & $\mathrm{C} 13-\mathrm{C} 12-\mathrm{H} 12 \mathrm{~B}$ & 117.7 \\
\hline $\mathrm{C} 3-\mathrm{C} 4-\mathrm{C} 7$ & $121.3(2)$ & $\mathrm{C} 11-\mathrm{C} 13-\mathrm{H} 13 \mathrm{~A}$ & 117.7 \\
\hline $\mathrm{C} 5-\mathrm{C} 4-\mathrm{C} 3$ & $117.5(2)$ & $\mathrm{C} 11-\mathrm{C} 13-\mathrm{H} 13 \mathrm{~B}$ & 117.7 \\
\hline $\mathrm{C} 5-\mathrm{C} 4-\mathrm{C} 7$ & $121.2(2)$ & $\mathrm{C} 12-\mathrm{C} 13-\mathrm{C} 11$ & $60.28(14)$ \\
\hline $\mathrm{C} 4-\mathrm{C} 5-\mathrm{H} 5$ & 119.2 & $\mathrm{C} 12-\mathrm{C} 13-\mathrm{H} 13 \mathrm{~A}$ & 117.7 \\
\hline $\mathrm{C} 6-\mathrm{C} 5-\mathrm{C} 4$ & $121.7(2)$ & $\mathrm{C} 12-\mathrm{C} 13-\mathrm{H} 13 \mathrm{~B}$ & 117.7 \\
\hline $\mathrm{C} 6-\mathrm{C} 5-\mathrm{H} 5$ & 119.2 & $\mathrm{H} 13 \mathrm{~A}-\mathrm{C} 13-\mathrm{H} 13 \mathrm{~B}$ & 114.9 \\
\hline $\mathrm{C} 1-\mathrm{C} 6-\mathrm{H} 6$ & 120.2 & $\mathrm{~N} 4-\mathrm{C} 14-\mathrm{H} 14 \mathrm{~A}$ & 109.5 \\
\hline $\mathrm{C} 5-\mathrm{C} 6-\mathrm{C} 1$ & $119.6(2)$ & $\mathrm{N} 4-\mathrm{C} 14-\mathrm{H} 14 \mathrm{~B}$ & 109.5 \\
\hline $\mathrm{C} 5-\mathrm{C} 6-\mathrm{H} 6$ & 120.2 & $\mathrm{~N} 4-\mathrm{C} 14-\mathrm{C} 15$ & $110.52(19)$ \\
\hline $\mathrm{C} 4-\mathrm{C} 7-\mathrm{H} 7 \mathrm{~A}$ & 109.5 & $\mathrm{H} 14 \mathrm{~A}-\mathrm{C} 14-\mathrm{H} 14 \mathrm{~B}$ & 108.1 \\
\hline $\mathrm{C} 4-\mathrm{C} 7-\mathrm{H} 7 \mathrm{~B}$ & 109.5 & $\mathrm{C} 15-\mathrm{C} 14-\mathrm{H} 14 \mathrm{~A}$ & 109.5 \\
\hline $\mathrm{C} 4-\mathrm{C} 7-\mathrm{H} 7 \mathrm{C}$ & 109.5 & $\mathrm{C} 15-\mathrm{C} 14-\mathrm{H} 14 \mathrm{~B}$ & 109.5 \\
\hline $\mathrm{H} 7 \mathrm{~A}-\mathrm{C} 7-\mathrm{H} 7 \mathrm{~B}$ & 109.5 & $\mathrm{O} 2-\mathrm{C} 15-\mathrm{C} 14$ & $109.4(2)$ \\
\hline $\mathrm{H} 7 \mathrm{~A}-\mathrm{C} 7-\mathrm{H} 7 \mathrm{C}$ & 109.5 & $\mathrm{O} 2-\mathrm{C} 15-\mathrm{H} 15 \mathrm{~A}$ & 109.8 \\
\hline $\mathrm{H} 7 \mathrm{~B}-\mathrm{C} 7-\mathrm{H} 7 \mathrm{C}$ & 109.5 & $\mathrm{O} 2-\mathrm{C} 15-\mathrm{H} 15 \mathrm{~B}$ & 109.8 \\
\hline $\mathrm{N} 1-\mathrm{C} 8-\mathrm{C} 9$ & $103.70(15)$ & $\mathrm{C} 14-\mathrm{C} 15-\mathrm{H} 15 \mathrm{~A}$ & 109.8 \\
\hline $\mathrm{N} 1-\mathrm{C} 8-\mathrm{C} 11$ & $124.99(17)$ & $\mathrm{C} 14-\mathrm{C} 15-\mathrm{H} 15 \mathrm{~B}$ & 109.8 \\
\hline $\mathrm{C} 9-\mathrm{C} 8-\mathrm{C} 11$ & $131.29(17)$ & $\mathrm{H} 15 \mathrm{~A}-\mathrm{C} 15-\mathrm{H} 15 \mathrm{~B}$ & 108.3 \\
\hline $\mathrm{N} 1-\mathrm{N} 2-\mathrm{N} 3-\mathrm{C} 9$ & $-0.6(2)$ & $\mathrm{C} 2-\mathrm{C} 3-\mathrm{C} 4-\mathrm{C} 5$ & $0.0(3)$ \\
\hline $\mathrm{N} 1-\mathrm{C} 1-\mathrm{C} 2-\mathrm{C} 3$ & $179.09(16)$ & $\mathrm{C} 2-\mathrm{C} 3-\mathrm{C} 4-\mathrm{C} 7$ & $-179.8(2)$ \\
\hline $\mathrm{N} 1-\mathrm{C} 1-\mathrm{C} 6-\mathrm{C} 5$ & $-178.65(17)$ & $\mathrm{C} 3-\mathrm{C} 4-\mathrm{C} 5-\mathrm{C} 6$ & $0.5(3)$ \\
\hline $\mathrm{N} 1-\mathrm{C} 8-\mathrm{C} 9-\mathrm{N} 3$ & $-1.0(2)$ & $\mathrm{C} 4-\mathrm{C} 5-\mathrm{C} 6-\mathrm{C} 1$ & $0.0(3)$ \\
\hline $\mathrm{N} 1-\mathrm{C} 8-\mathrm{C} 9-\mathrm{C} 10$ & $178.57(19)$ & $\mathrm{C} 6-\mathrm{C} 1-\mathrm{C} 2-\mathrm{C} 3$ & $1.5(3)$ \\
\hline
\end{tabular}




$125.2(2)$
$55.1(3)$
$-146.14(17)$
$31.5(2)$
$0.61(19)$
$-177.98(16)$
$1.0(2)$
$-178.57(17)$
$168.7(2)$
$-12.2(3)$
$-58.8(3)$
$-179.30(16)$
$179.77(18)$
$1.2(3)$
$-1.0(3)$
$-1.0(3)$

$\mathrm{C} 7-\mathrm{C} 4-\mathrm{C} 5-\mathrm{C} 6$

$\mathrm{C} 8-\mathrm{N} 1-\mathrm{N} 2-\mathrm{N} 3$

$\mathrm{C} 8-\mathrm{N} 1-\mathrm{C} 1-\mathrm{C} 2$

$\mathrm{C} 8-\mathrm{N} 1-\mathrm{C} 1-\mathrm{C} 6$

$\mathrm{C} 8-\mathrm{C} 9-\mathrm{C} 10-\mathrm{O} 1$

$\mathrm{C} 8-\mathrm{C} 9-\mathrm{C} 10-\mathrm{N} 4$

$\mathrm{C} 8-\mathrm{C} 11-\mathrm{C} 12-\mathrm{C} 13$

$\mathrm{C} 8-\mathrm{C} 11-\mathrm{C} 13-\mathrm{C} 12$

C9-C8-C11- 12

$\mathrm{C} 9-\mathrm{C} 8-\mathrm{C} 11-\mathrm{C} 13$

$\mathrm{C} 10-\mathrm{N} 4-\mathrm{C} 14-\mathrm{C} 15$

$\mathrm{C} 11-\mathrm{C} 8-\mathrm{C} 9-\mathrm{N} 3$

$\mathrm{C} 11-\mathrm{C} 8-\mathrm{C} 9-\mathrm{C} 10$

C14-N4- $10-\mathrm{O} 1$

$\mathrm{C} 14-\mathrm{N} 4-\mathrm{C} 10-\mathrm{C} 9$
$-179.7(2)$

$0.0(2)$

34.7 (3)

$-147.61(19)$

-10.8 (3)

$168.3(2)$

$-111.0(2)$

$108.5(2)$

$-53.0(3)$

$-123.1(2)$

$-141.9(2)$

$177.49(18)$

$-3.0(3)$

$-1.9(3)$

$179.0(2)$

Hydrogen-bond geometry $\left(A,{ }^{\circ}\right)$

\begin{tabular}{lllll}
\hline$D-\mathrm{H} \cdots A$ & $D-\mathrm{H}$ & $\mathrm{H} \cdots A$ & $D \cdots A$ & $D-\mathrm{H} \cdots A$ \\
\hline $\mathrm{O} 2-\mathrm{H} 2 \cdots \mathrm{O} 1^{\mathrm{i}}$ & $0.95(3)$ & $1.78(3)$ & $2.734(2)$ & $177(3)$ \\
$\mathrm{C} 11-\mathrm{H} 11 \cdots \mathrm{N} 3^{\mathrm{ii}}$ & 0.98 & 2.61 & $3.391(2)$ & 137 \\
$\mathrm{C} 5-\mathrm{H} 5 \cdots \mathrm{O} 2^{\mathrm{iii}}$ & 0.93 & 2.66 & $3.564(3)$ & 164 \\
\hline
\end{tabular}

Symmetry codes: (i) $x,-y+1 / 2, z-1 / 2$; (ii) $x,-y+1 / 2, z+1 / 2$; (iii) $-x+1,-y+1,-z+1$. 